\title{
Electrical field profile and doping in planar lead halide perovskite solar cells
}

\author{
Antonio Guerrero, ${ }^{1}$ Emilio J. Juarez-Perez, ${ }^{1}$ Juan Bisquert, ${ }^{1,2}$ Ivan Mora-Sero, ${ }^{1}$ \\ and Germà Garcia-Belmonte ${ }^{1, a)}$ \\ ${ }^{1}$ Photovoltaic and Optoelectronic Devices Group, Departament de Física, Universitat Jaume I, \\ ES-12071 Castelló, Spain \\ ${ }^{2}$ Department of Chemistry, Faculty of Science, King Abdulaziz University, Jeddah 21589, Saudi Arabia
}

(Received 28 July 2014; accepted 18 September 2014; published online 29 September 2014)

\begin{abstract}
Hybrid lead halide perovskites (PVKs) have emerged as novel materials for photovoltaics and have rapidly reached very large solar to electricity power conversion efficiencies. As occurring with other kind of solar technologies establishing the working energy-band diagram constitutes a primary goal for device physics analysis. Here, the macroscopic electrical field distribution is experimentally determined using capacitance-voltage and Kelvin probe techniques. Planar structures comprising $\mathrm{CH}_{3} \mathrm{NH}_{3} \mathrm{PbI}_{3-\mathrm{x}} \mathrm{Cl}_{\mathrm{x}} \mathrm{PVK}$ exhibit $p$-doping character and form a $p$ - $n$ heterojunction with $n$-doped $\mathrm{TiO}_{2}$ compact layers. Depletion width at equilibrium within the PVK bulk has an extent about $300 \mathrm{~nm}$ (approximately half of the layer thickness), leaving as a consequence a significant neutral zone towards the anode contact. Charge collection properties are then accessible relying on the relative weight that diffusion and drift have as carrier transport driven forces. (C) 2014 AIP Publishing LLC. [http://dx.doi.org/10.1063/1.4896779]
\end{abstract}

Recently, hybrid lead halide perovskites (PVKs) have reached very large solar to electricity power conversion efficiencies, ${ }^{1,2}$ with efficiencies $16 \% .^{3}$ In the last two years, many different configurations have been analyzed varying cell configuration, selective contacts, and even the kind of perovskite utilized. ${ }^{1,2}$ Despite this broad range of variations, probably the most extensively studied has been the $\mathrm{CH}_{3} \mathrm{NH}_{3} \mathrm{PbI}_{3}$ perovskite (or its analogous but using chlorine precursor: $\mathrm{CH}_{3} \mathrm{NH}_{3} \mathrm{PbI}_{3-\mathrm{x}} \mathrm{Cl}_{\mathrm{x}}$ ) as absorber materials, in combination with electron $\left(\mathrm{TiO}_{2}\right)$ and hole (spiro-OMeTad) selective contacts. The organic cation $\mathrm{CH}_{3} \mathrm{NH}_{3}{ }^{+}$is mainly responsible for the structural stability of the PVK, while the electronic properties are largely determined by metal and halide hybridized orbitals. ${ }^{4-6}$ In spite of the spectacular advances in cell efficiency, many aspects of this system are not completely understood. One central piece of information for understanding the photovoltaic operation of these solar cells is the energy-band diagram. In general, the band diagram provides key knowledge of several aspects intervening into the working mechanisms of solar cells, namely, the spatial distribution of the macroscopic electrical field within the absorber layer determining charge collection properties; the direction of electrical field and dipole layer at contacts; the local density of electron and hole carrier at each point of the device. Here, we report on characteristic energy features of PVK solar cells based on the combined use of capacitancevoltage $(C-V)$ and Kelvin probe force microscopy (KPFM).

Because of the energetic offset between work functions of the absorber and each contact material, it is expected the formation of a built-in voltage $V_{b i}$ in equilibrium conditions. There are several ways to accommodate $V_{b i}$ depending on the electrostatic characteristics of the bulk materials and interfaces. For undoped absorber materials a rather constant

\footnotetext{
${ }^{\text {a) }}$ Author to whom correspondence should be addressed. Electronic mail: garciag@uji.es. Tel.: +34 964 387538. Fax: +34 964729218.
}

electrical field along the whole bulk is expected as occurring for $p-i-n$ structures. However, doping alters the electrical field distribution by the presence of charged impurities or defects of structural or chemical origin introduced during device processing. Homogeneous defect density gives rise to quadratic band bending profiles, which concentrate the electrical field in the vicinity of the contact and leave large quasi-neutral zones within the bulk layer. Charge transport of minority carriers throughout quasi-neutral zones occurs by diffusion while drift governs the carrier motion within band bending regions. Then collection efficiency is governed by the electrical field distribution which might exhibit several profiles depending on the doping density and applied voltage.

It has been recently suggested that perovskite-based solar cells either with flat or mesoporous electron transport material operate as a $p$-i-n-type structure. ${ }^{7}$ Electrical field distribution has been addressed in cells comprising mesoporous $\mathrm{TiO}_{2}$ by capacitive techniques with electron transporting layer electrically modeled as a homogeneous layer. ${ }^{8}$ Spatially resolved electron beam-induced current has been reported to support that carriers are efficiently collected throughout the whole absorber layer. ${ }^{9}$ Although high electron beam-induced signal would indicate rather good transport properties, there is no direct determination of the electrical field distribution that allows accounting for the relative weight that diffusion and drift have as carrier transport driven forces. Here, we use planar structures of the type $\mathrm{FTO} / \mathrm{TiO}_{2} / \mathrm{PVK} / 2,2^{\prime}, 7,7^{\prime}$ tetrakis(N,N-di-p-methoxyphenylamine)-9,9-spirobifluorene(spiro-OMeTad)/Au to show that $\mathrm{CH}_{3} \mathrm{NH}_{3} \mathrm{PbI}_{3-\mathrm{x}} \mathrm{Cl}_{\mathrm{x}} \mathrm{PVK}$ exhibits $p$-doping character and forms a $p$ - $n$ heterojunction with $n$-doped $\mathrm{TiO}_{2}$ compact layers. Planar configuration has been chose as it offers a simpler configuration in comparison with samples with nanoporous scaffold, and the reported effect can be just attributed to PVK. We have observed that depletion width at equilibrium within the PVK bulk has an extent about $300 \mathrm{~nm}$ (approximately half of the layer 
thickness). When cells are constructed without the holetransporting layer spiro-OMeTad the amount of PVK doping is dramatically reduced yielding full depletion devices even at voltages below $0.5 \mathrm{~V}$ forward bias as in a $p-i-n$ structure.

All the studied cells of structure $\mathrm{FTO} / \mathrm{TiO}_{2} / \mathrm{PVK} /$ spiroOMeTad/Au were prepared ${ }^{7}$ over FTO (Pilkington TEC15, 15 $\Omega / \mathrm{sq}$ resistance), which were previously etched with zinc powder and $\mathrm{HCl}(2 \mathrm{M})$. The FTOs substrates were cleaned with soap (Hellmanex), deionized water, and ethanol, followed by sonication in a mixture acetone/isopropanol $(\mathrm{v} / \mathrm{v}=1: 1)$. Before the deposition of electron selective contacts, substrates were treated in a UV-O ${ }_{3}$ chamber for $30 \mathrm{~min}$. The $\mathrm{TiO}_{2}$ compact layer was deposited by spin-coating a mildly acidic solution of titanium isopropoxide in ethanol, and heated at $500{ }^{\circ} \mathrm{C}$ for $30 \mathrm{~min}$. The mixed halide perovskite was prepared by spin coating $100 \mu \mathrm{l}$ of perovskite precursor, $40 \%$ (w/w) DMF solution of $\mathrm{CH}_{3} \mathrm{NH}_{3} \mathrm{I}$, and $\mathrm{PbCl}_{2}$ (3:1 molar ratio), onto flat $\mathrm{TiO}_{2}$ substrates at $2000 \mathrm{rpm}$ for $60 \mathrm{~s}$. Then, films were placed on a heated furnace at $100^{\circ} \mathrm{C}$ under an air stream. The perovskiteadsorbed films were covered with a hole transporting material (HTM) layer by spin coating at $4000 \mathrm{rpm}$ for $30 \mathrm{~s}$ outside of the glovebox. The HTM recipe was prepared dissolving $72.3 \mathrm{mg}$ of spiro-OMeTad, $28.8 \mu \mathrm{l}$ of 4-tert-butylpyridine, and $17.5 \mu \mathrm{l}$ of a stock solution of $520 \mathrm{mg} / \mathrm{ml}$ lithium bis-(trifluoromethylsulfonyl)imide in acetonitrile in $1 \mathrm{ml}$ of chlorobenzene. Other set of cells were prepared without HTM. Finally, $60 \mathrm{~nm}$ of gold was thermally evaporated in the vacuum chamber on top of the device to form the electrode contacts. An active electrode area of $0.224 \mathrm{~cm}^{2}$ per pixel is defined by the FTO and the Au contacts. The device fabrication was carried out under controlled atmospheric conditions and a humidity of $<0.5 \mathrm{ppm}$, and the HTM layer deposition was carried out at room conditions. Current density-voltage, $J-V$, curves were recorded under AM1.5 $100 \mathrm{~mW} \mathrm{~cm}{ }^{-2}$ simulated sunlight (ABET Technologies Sun 2000) previously calibrated with an NREL-calibrated Si solar cell. The $J-V$ measurements were performed using a $0.08 \mathrm{~cm}^{2}$ mask. Impedance spectroscopy measurements were carried out with a PGSTAT-30 from Autolab, in dark conditions and at different forward biases, by applying a $20 \mathrm{mV}$ AC voltage perturbation over the constant forward DC applied bias, between 0 and $1 \mathrm{~V}$, with the frequency ranging between $1 \mathrm{MHz}$ and $0.05 \mathrm{~Hz}$. Capacitance values are obtained at frequencies of $600 \mathrm{~Hz}$ as a plateau is observed in the capacitance vs frequency plot at frequencies in the range of $100 \mathrm{~Hz}-10 \mathrm{kHz}$. KPFM was performed using a Nano-Observer Atomic Force Microscope (Concept Scientific Instruments).

Typical $J-V$ curves are presented in Fig. 1 for cells with and without spiro-OMeTad as HTM. Figure 2(a) shows energy levels of $\mathrm{TiO}_{2}$ and PVK layers. $\mathrm{TiO}_{2}$ forms a good electron contact that blocks hole extraction because of the deep laying valence band. As next discussed the measured built-in voltage $V_{b i}$ at the $\mathrm{TiO}_{2}$ side is always quite large $(\sim 1 \mathrm{~V})$ indicating that after equilibration the device accommodates a large potential drop. The model assumes an ideal anisotype heterojunction formed at the contact between the $\mathrm{TiO}_{2}$ and perovskite flat layers. $V_{b i}$ corresponds to the difference in work function (Fermi levels) between the two sides. Since $V_{b i}$ is large, one possibility is having a $p$ - $n$ (perovskite$\mathrm{TiO}_{2}$ ) type heterojunction. Other combinations as $n-n$ isotype

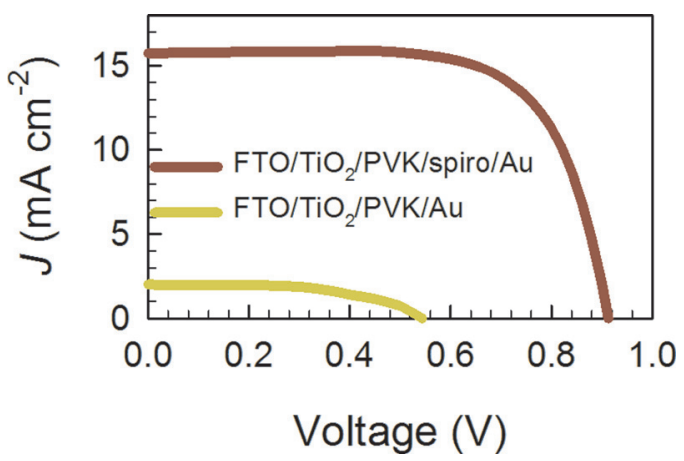

FIG. 1. Typical $J-V$ curves of the solar cells with structures $\mathrm{FTO} / \mathrm{TiO}_{2} /$ $\mathrm{CH}_{3} \mathrm{NH}_{3} \mathrm{PbI}_{3-\mathrm{x}} \mathrm{Cl}_{\mathrm{x}} /$ spiro-OMeTad/Au and $\mathrm{FTO} / \mathrm{TiO}_{2} / \mathrm{CH}_{3} \mathrm{NH}_{3} \mathrm{PbI}_{3-\mathrm{x}} \mathrm{Cl}_{\mathrm{x}} /$ $\mathrm{Au}$.

function can be ruled out as lower $V_{b i}$ values would result. The basic band diagram is displayed in Fig. 2(b) which does not consider interface traps forming a dipole contact layer. Interface dipoles can be discarded as they reduce the flatband voltage accommodating part of the work function offset. The diagram is still valid provided that the PVK work function is placed deeper than the $\mathrm{TiO}_{2}$ work function, irrespective of the $n$ or $p$ character. ${ }^{10}$

Depletion zones are formed both at $p$ and $n$ side of the junction, $w_{\mathrm{PVK}}$ and $w_{\mathrm{TiO}_{2}}$ which are modulated by the applied voltage provided that FTO and spiro-OMeTad/Au form good ohmic contacts. Perovskite presents $p$-doped character with an acceptor defect density $N_{\mathrm{PVK}}$, while $\mathrm{TiO}_{2}$ is $n$-doped with donor defect density $N_{\mathrm{TiO}_{2}}$, which will be considered as position-independent. The total built-in voltage is divided (a)

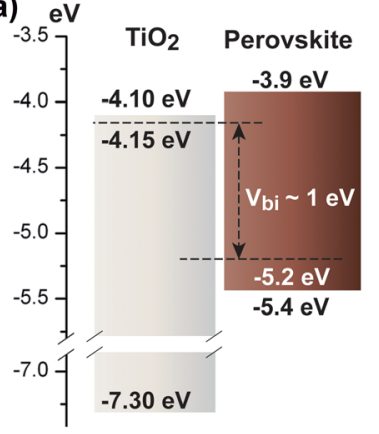

(c)

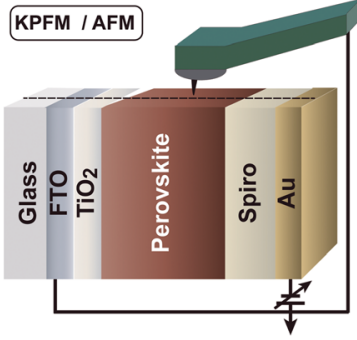

(b)

(d)

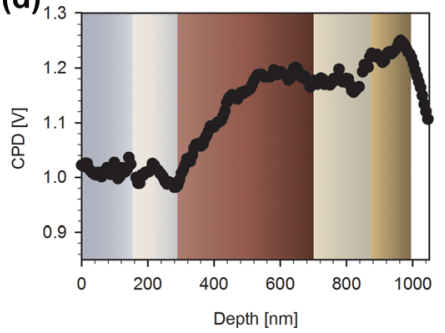

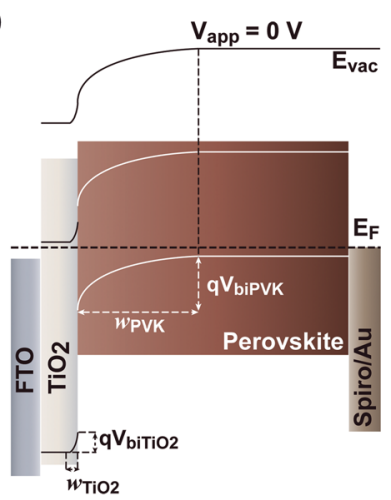

FIG. 2. (a) Illustrative energy diagram of the separate materials, $\mathrm{TiO}_{2}$ and PVK. The position of the respective workfunction is based on the doping level encountered. A built-in voltage approximately equal to $1 \mathrm{eV}$ is expected. (b) Band diagram in equilibrium showing the formation of a $p-n$ (perovskite- $\mathrm{TiO}_{2}$ ) type heterojunction. The extension of the respective depletion zones and built-in voltages are indicated at each side. (c) Drawing of the Kelvin probe force microscopy signaling the lateral path over which contact potential difference is monitored CPD (d). Average values over several tracks are displayed. 


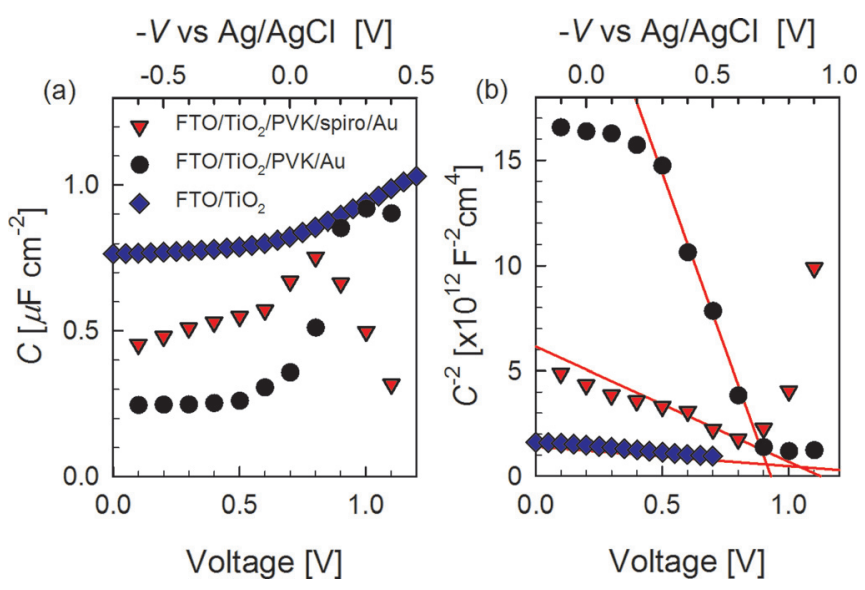

FIG. 3. (a) Capacitance vs voltage response and (b) Mott-Schottky analysis of devices described in this work. For $\mathrm{FTO} / \mathrm{TiO}_{2}$ an electrochemical threeelectrode setup is used with $\mathrm{Ag} / \mathrm{AgCl}$ as reference electrode.

into two parts $V_{b i}=V_{b i \mathrm{PVK}}+V_{b i \mathrm{TiO}_{2}}$ as indicated in Fig. 2(b). The depletion widths and the junction capacitance per unit area $C$ can be obtained by solving the Poisson's equation with the boundary condition of the continuity of the electrical displacement. Because contact equilibration is assumed the formation of depletion zones entails static values for the permittivity of each material $\varepsilon_{\mathrm{PVK}}$ and $\varepsilon_{\mathrm{TiO}_{2}}$, respectively ${ }^{11}$

$$
\begin{aligned}
& w_{\mathrm{PVK}}=\sqrt{\frac{2 N_{\mathrm{TiO}_{2}} \varepsilon_{\mathrm{PVK}} \varepsilon_{\mathrm{TiO}_{2}}\left(V_{b i}-V\right)}{q N_{\mathrm{PVK}}\left(\varepsilon_{\mathrm{PVK}} N_{\mathrm{PVK}}+\varepsilon_{\mathrm{TiO}_{2}} N_{\left.\mathrm{TiO}_{2}\right)}\right.}}, \\
& w_{\mathrm{TiO}_{2}}=\sqrt{\frac{2 N_{\mathrm{PVK} \varepsilon_{\mathrm{PVK}} \varepsilon_{\mathrm{TiO}_{2}}\left(V_{b i}-V\right)}^{q N_{\mathrm{TiO}_{2}}\left(\varepsilon_{\mathrm{PVK}} N_{\mathrm{PVK}}+\varepsilon_{\mathrm{TiO}_{2}} N_{\mathrm{TiO}_{2}}\right)}}{C=}} \\
& \frac{q N_{\mathrm{PVK}} N_{\mathrm{TiO}_{2}} \varepsilon_{\mathrm{PVK}} \varepsilon_{\mathrm{TiO}_{2}}}{2\left(\varepsilon_{\mathrm{PVK}} N_{\mathrm{PVK}}+\varepsilon_{\mathrm{TiO}_{2}} N_{\mathrm{TiO}_{2}}\right)\left(V_{b i}-V\right)}
\end{aligned}
$$

This last expression is a version of the well-known MottSchottky relation $C^{-2} \propto V$ with a slope that depends on the doping level and permittivity of the two semiconductor junction sides. The relative voltage drop at each side of the junction at $V=0$ is ${ }^{11}$

$$
\frac{V_{b i \mathrm{PVK}}}{V_{b i \mathrm{TiO}_{2}}}=\frac{N_{\mathrm{TiO}_{2}} \varepsilon_{\mathrm{TiO}_{2}}}{N_{\mathrm{PVK}} \varepsilon_{\mathrm{PVK}}} .
$$

By examining Eq. (2), one can infer that the Mott-Schottky analysis gives mixed information in which contribute both semiconductor sides. ${ }^{8}$ In order to separate the relative influence of each junction side, it is necessary to calculate one of them independently. Doping level of the $\mathrm{TiO}_{2}$ compact layer can be measured by employing an electrochemical three-electrode setup in which $\mathrm{FTO} / \mathrm{TiO}_{2}$ forms the working electrode. ${ }^{12}$ A suitable electrolyte plays the contacting role of the perovskite layer in solar cells. Capacitance measurements are shown in Fig. 3(a) represented as a function of the reference electrode $(\mathrm{Ag} / \mathrm{AgCl})$ voltage. The MottSchottky calculation in electrochemical configuration allows estimating $\mathrm{TiO}_{2}$ doping level using the well-known relationship $C^{-2}=2\left(V_{b i}-V\right) / q \varepsilon_{\mathrm{TiO}_{2}} N_{\mathrm{TiO}_{2}}$ see [Fig. 3(b)]. Relatively high doping levels are encountered which result in $N_{\mathrm{TiO}_{2}} \approx 6.2 \times 10^{18} \mathrm{~cm}^{-3}$ (see Table I). Here, it is assumed that the FTO layer presents a much larger doping density because of its high conductivity. $\mathrm{TiO}_{2}$ doping value entails a Fermi level position approximately equal to $50 \mathrm{meV}$ below the conduction band. The electrochemical setup cannot allow straightforwardly determining the semiconductor permittivity because of the influence of the double-layer capacitors formed at the solid/liquid junction. Instead the $\mathrm{TiO}_{2}$ permittivity can be easily calculated from the high-frequency capacitance value measured in a FTO/ $\mathrm{TiO}_{2} / \mathrm{Au}$ diode in reverse bias. It was checked that the permittivity derived from the geometrical capacitance is thickness-independent (see supplementary material). ${ }^{13}$ The relative permittivity results in $\varepsilon_{r} \mathrm{TiO}_{2}=20 \pm 2$ in good agreement with previous measurements signaling the variation of the $\mathrm{TiO}_{2}$ permittivity upon the processing conditions and film thickness. ${ }^{14,15}$

Using this information, it is feasible to explore the doping level of the lead halide PVK forming the absorber layer of a high efficiency solar cell. Capacitance values are carefully selected from the capacitance spectra at a frequency at which a plateau is observed. This occurs in the intermediatefrequency range $(\sim 1 \mathrm{kHz}) .{ }^{13}$ At high-frequencies the effect of the series resistance yields lower than expected values, while in the low-frequency range large distortions caused by the recently reported giant permittivity could dominate. ${ }^{16} \mathrm{~A}$ representative capacitance and Mott-Schottky plot are displayed in Fig. 3. As latter explained $\varepsilon_{\mathrm{PVK}}$ is estimated under conditions of full depletion. For the standard structure FTO/ $\mathrm{TiO}_{2} / \mathrm{PVK} /$ spiro-OMeTad/Au, the doping level is found to be about $N_{\mathrm{PVK}} \approx 1.8 \times 10^{17} \mathrm{~cm}^{-3}$, smaller than the $\mathrm{TiO}_{2}$ doping level. That moves up the Fermi level around $200 \mathrm{meV}$. Using these doping values one would expect a total built-in voltage approaching $1 \mathrm{~V}$, in good agreement with the experimentally measured $V_{b i}$ as illustrated in Fig. 2(a).

The previous calculations entail that the work function offset encountered between $\mathrm{TiO}_{2}$ and perovskite is accommodated in the vicinity of the heterojunction as schematically drawn in Fig. 2(b). The respective depletion widths are calcu-

\begin{tabular}{|c|c|c|c|c|c|c|}
\hline Device structure & $J_{\mathrm{sc}}\left(\mathrm{mA} \mathrm{cm}{ }^{-2}\right)$ & $V_{\mathrm{oc}}(\mathrm{mV})$ & $\mathrm{FF}(\%)$ & $\operatorname{PCE}(\%)$ & $N\left(\times 10^{16}\right)\left(\mathrm{cm}^{-3}\right)$ & $V_{b i}(\mathrm{~V})$ \\
\hline $\mathrm{FTO} \mathrm{TiO}_{2}$ & $\ldots$ & $\ldots$ & $\ldots$ & $\ldots$ & 620 & $-1.25^{\mathrm{a}}$ \\
\hline $\mathrm{FTO} \mathrm{TiO}_{2}$-PVK-spiro-Au & 15.77 & 913 & 70 & 10.0 & 18.0 & 1.19 \\
\hline FTO-TiO $2-\mathrm{PVK}-\mathrm{Au}$ & 2.02 & 544 & 54 & 0.6 & 3.2 & 0.94 \\
\hline
\end{tabular}
lated using Eq. (1), and each built-in voltage calculation at

TABLE I. Photovoltaic parameters of $\mathrm{CH}_{3} \mathrm{NH}_{3} \mathrm{PbI}_{3-\mathrm{x}} \mathrm{Cl}_{\mathrm{x}}$ PVK-based devices measured under 1 sun illumination using different processing conditions. Doping density $N$ of $\mathrm{TiO}_{2}$ and PVK layers and built-in voltage $V_{b i}$ extracted from capacitance-voltage measurements in dark conditions.

${ }^{\mathrm{a}}$ Voltage vs. $\mathrm{Ag} / \mathrm{AgCl}$. 
zero bias relies on Eq. (3). Interestingly, the depletion zone only extents a few nm within the bulk of the $\mathrm{TiO}_{2}$ layer because of the relatively high doping level. ${ }^{17}$ In the PVK side the depletion zone reaches around $300 \mathrm{~nm}$, approximately half of the layer thickness $(\sim 450 \mathrm{~nm})$. Hence, the absorber layer is not fully depleted at zero bias existing as a consequence a significant neutral zone towards the anode contact. More importantly, the electrical field is restricted to the space-charge zone made up by immobile acceptor defects near the electron contact. In the rest of the bulk (neutral zone) free hole carriers efficiently shield electrical fields. Since the built-in voltage at the PVK side is relatively large $V_{b i \mathrm{PVK}}$ $=0.89 \mathrm{~V}$, it occurs that the Fermi level crosses midgap energies yielding a thin inversion layer near the interface.

To support the electrical field profile derived from $C-V$ techniques, alternative measurements have been carried out using KPFM methods [see Fig. 2(c) and supplementary material for details]. ${ }^{13}$ KPFM allows investigation of the cross-sectional potential distribution in complete devices. Very recently, the enormous potential for characterization of Kelvin probe has been employed to investigate the role of selective contacts in perovskite solar cells, exploring the surface of the samples. ${ }^{18}$ Here, KPFM has been employed for the characterization of the cross-sectional potential distribution in complete devices. Fig. 2(d) displays contact potential difference average values over several lateral tracks. It is observed that a potential step in the PVK bulk contacting the $\mathrm{TiO}_{2}$ layer extends about $300 \mathrm{~nm}$ inside the absorber bulk, while a flat profile appears in the rest of the layer. KPFM is not able to resolve with sufficient spatial accuracy the voltage drop in the $\mathrm{TiO}_{2}$ side occurring in just $5 \mathrm{~nm}$. It should be noted that the average voltage step measured by means of $\operatorname{KPFM}(\sim 0.2 \mathrm{~V})$ is below the internal $V_{b i}$ calculated from $C-V$ techniques. A reason for this discrepancy might be found in the different measuring setup that each technique uses. For $C-V$ the junction capacitance is extracted which scales with the active surface area. On the contrary, KPFM allows determining the contact potential following a lateral, external cross-section path as drawn in Fig. 2(c). Here potential distribution not only depends on transversal, bulk properties, but it is certainly distorted by longitudinal effects. As previously addressed by Ottinger et al. ${ }^{19}$ there is a difference between the thickness-dependent surface potential and the bulk potential caused by an inherent potential drop at the substrate/semiconductor interface. Specially, doped semiconductors can show deviations that are not negligible. In any case, potential profile derived from KPFM qualitative agrees with that extracted from Mott-Schottky analysis. Despite the inherent limitation in spatial resolution KPFM, it is found again that the electrical field is confined near the cathode with typical size of $300 \mathrm{~nm}$, with a bulky region of the absorber to be nearly field-free. The electrical field profiles derived from our experiments allows drawing a featured picture concerning the driving force for carrier transport, which is governed either by diffusion or drift mechanisms depending on voltage-dependent PVK depletion zone extent.

As commented before, ionized defects play a crucial role in establishing space-charge regions which ultimately withstand the band bending at the contact between absorber and selective layer. In order to provide deeper insight into the origin of PVK impurities responsible for depletion zones, different device processing conditions have been checked and analyzed by $C-V$ techniques. Devices not including the hole transporting spiro-OMeTad layer have been prepared by evaporating Au contacts directly onto PVK layers. Although these devices exhibit lower power conversion efficiency than those obtained with the standard structure (see Table I), it permits to discern how preparation conditions might alter potential distributions. Fig. 3 shows that the Mott-Schottky linear relation is still observed although with larger slope. Lower defect density level is then found when spiro-OMeTad layer is suppressed, which results in $N_{\mathrm{PVK}} \approx 3.2 \times 10^{16} \mathrm{~cm}^{-3}$ (see Table I). As observed in Fig. 3(a), a plateau in capacitance occurs at voltages below $0.4 \mathrm{~V}$. This constant capacitance corresponds to the capacitance $C=0.24 \mu \mathrm{F} \mathrm{cm}^{-2}$ that can be related to the series combination of the $\mathrm{TiO}_{2}$ layer and PVK layer capacitances, $C^{-1}=C_{\mathrm{TiO}_{2}}^{-1}+C_{\mathrm{PVK}}^{-1}$. Since $C_{\mathrm{TiO} 2}$ can be independently measured in the $\mathrm{FTO} / \mathrm{TiO}_{2} / \mathrm{Au}$ diode in reverse bias, perovskite relative permittivity $\left(\varepsilon_{r \mathrm{PVK}}=230 \pm 10\right)$ is extracted in full-depletion conditions taking into account the absorber thickness of $\sim 200 \mathrm{~nm}$ in the cell $\mathrm{FTO} / \mathrm{TiO}_{2} / \mathrm{PVK} / \mathrm{Au}$. In contrast, it has been recently pointed out that $\varepsilon_{r} \mathrm{PVK}$ is highly dependent on the density of free carriers, either intrinsic or photogenerated. ${ }^{16}$ It was found an unprecedented behavior in which the low-frequency permittivity measured below $1 \mathrm{~Hz}$ increases under illumination or by charge injection. $\varepsilon_{r} \mathrm{PVK}$ increases nearly linearly with the illumination intensity from $\sim 2000$ in the dark up to $10^{6}$ under 1 sun. The photogenerated carriers in a deformable polar medium exhibit polaronic features, and their slow motion, which drags the lattice distortion, is denoted polaron hopping. An excess of localized charge, with a relative ability to move under external or thermal force, would modify the ionic bonding balance of the unit cell and cause local distortion. This process involves the macroscopic rearrangement of dipolar domains, and it will influence the long-range carrier transport. Indeed, it can be observed $^{16}$ that in the low frequency conductivity decreases by 2 orders of magnitude with respect to the intermediate frequency $(1 \mathrm{kHz})$ conductivity. The junction capacitance, which is measured at much higher frequencies $(600 \mathrm{~Hz}$ as explained in Ref. 13), is related to depletion zones which are built by the presence of immobile charged impurities with majority carriers removed. This explains why $\varepsilon_{r} \mathrm{PVK}$ extracted from the Mott-Schottky analysis is only 230. On the contrary, low-frequency $(1 \mathrm{~Hz})$ capacitances is influenced by the response of quasi-neutral zones in which the carrier density is not negligible then enlarging $\varepsilon_{r \mathrm{PVK}}$. It is then consistent that the static permittivity found here results below typical values of $\sim 2000$ observed at low frequencies in the dark. ${ }^{16}$ More importantly, for this configuration PVK layer undergoes full depletion for $V<0.4 \mathrm{~V}$ recovering the electrical field distribution extending over the whole bulk as expected for an intrinsic absorber layer.

The preceding experimental data conclude that the electrical field profile along the PVK bulk is dictated by the amount of defects responsible for the perovskite doping. A simple calculation using Eq. (1a) situates the doping level of $N_{\mathrm{PVK}} \approx 8.5 \times 10^{16} \mathrm{~cm}^{-3}$ as the limit below which the occurrence of full-depletion is achievable at $0 \mathrm{~V}$-bias. It is 
interesting to note that the growth method can affect significantly the doping level., ${ }^{4,5}$ The presence and extent of electrical fields are known to change dramatically the charge collection efficiency by varying the relative weight of carrier drift and diffusion mechanisms on the overall transport driving force. Although more extended electrical fields are encountered when spiro-OMeTad layer is not deposited, the solar cell performance suffers from poor contact selectivity. It should be stressed here that precisely the inclusion of the spiro-OMeTad layer promotes carrier extraction at the contact, but at the same time it increases PVK doping. This might be related to the incorporation of light additives such as Li salts usually employed during layer processing and it is an interesting point to be explored in future work.

We thank financial support by MINECO of Spain under project (MAT2013-47192-C3-1-R), and ISIC/2012/008 Institute of Nanotechnologies for Clean Energies. We would like to express our gratitude to Dr. Francisco Martinez from Scientec for carrying out the KPFM measurements. SCIC services at UJI are acknowledged.

${ }^{1}$ N.-G. Park, J. Phys. Chem. Lett. 4, 2423 (2013).

${ }^{2}$ H. J. Snaith, J. Phys. Chem. Lett. 4, 3623 (2013).

${ }^{3}$ N. J. Jeon, J. H. Noh, Y. C. Kim, W. S. Yang, S. Ryu, and S. I. Seok, Nat. Mater. 13, 897 (2014).
${ }^{4}$ H. J. Snaith, A. Abate, J. M. Ball, G. E. Eperon, T. Leijtens, N. Kimberly, S. D. Stranks, J. T.-W. Wang, K. Wojciechowski, W. Zhang, and N. K. Noel, J. Phys. Chem. Lett. 5, 1511 (2014).

${ }^{5}$ W.-J. Yin, T. Shi, and Y. Yan, Appl. Phys. Lett. 104, 063903 (2014).

${ }^{6}$ J. Even, L. Pedesseau, J.-M. Jancu, and C. Katan, J. Phys. Chem. Lett. 4, 2999 (2013).

${ }^{7}$ J. M. Ball, M. M. Lee, A. Hey, and H. J. Snaith, Energy Environ. Sci. 6, 1739 (2013)

${ }^{8}$ W. A. Laban and L. Etgar, Energy Environ. Sci. 6, 3249 (2013).

${ }^{9}$ E. Edri, S. Kirmayer, A. Henning, S. Mukhopadhyay, K. Gartsman, Y. Rosenwaks, G. Hodes, and D. Cahen, Nano Lett. 14, 1000 (2014).

${ }^{10}$ J. Kim, S.-H. Lee, J. H. Lee, and K.-H. Hong, J. Phys. Chem. Lett. 5, 1312 (2014).

${ }^{11}$ S. M. Sze, Physics of Semiconductor Devices, 2nd ed. (John Wiley \& Sons, Inc., New York, 1981).

${ }^{12}$ L. Kavan, N. Tetreault, T. Moehl, and M. Grätzel, J. Phys. Chem. C 118, 16408 (2014).

${ }^{13}$ See supplementary material at http://dx.doi.org/10.1063/1.4896779 for experimental details.

${ }^{14}$ M. D. Stamate, Appl. Surf. Sci. 218, 318 (2003).

${ }^{15}$ V.-S. Dang, H. Parala, J. H. Kim, K. Xu, N. B. Srinivasan, E. Edengeiser, M. Havenith, A. D. Wieck, T. d. 1. Arcos, R. A. Fischer, and A. Devi, Phys. Status Solidi A 211, 416 (2014).

${ }^{16}$ E. J. Juarez-Perez, R. S. Sanchez, L. Badia, G. Garcia-Belmonte, Y. S. Kang, I. Mora-Sero, and J. Bisquert, J. Phys. Chem. Lett. 5, 2390-2394 (2014).

${ }^{17}$ K. Wojciechowski, M. Saliba, T. Leijtens, A. Abate, and H. Snaith, Energy Environ. Sci. 7, 1142 (2014).

${ }^{18}$ L. Barnea-Nehoshtan, S. Kirmayer, E. Edri, G. Hodes, and D. Cahen, J. Phys. Chem. Lett. 5, 2408 (2014).

${ }^{19}$ O. M. Ottinger, C. Melzer, and H. V. Seggern, J. Appl. Phys. 106, 023704 (2009). 